Research Article

\title{
Hederagenin Induces Apoptosis in Cisplatin-Resistant Head and Neck Cancer Cells by Inhibiting the Nrf2-ARE Antioxidant Pathway
}

\author{
Eun Hye Kim, ${ }^{1}$ Seungho Baek, ${ }^{2}$ Daiha Shin, ${ }^{1}$ Jaewang Lee, ${ }^{1}$ and Jong-Lyel Roh ${ }^{1}$ \\ ${ }^{1}$ Department of Otolaryngology, Asan Medical Center, University of Ulsan College of Medicine, Seoul, Republic of Korea \\ ${ }^{2}$ Department of Pathology, College of Korean Medicine, Woosuk University, Jeonju-si, Jeollabuk-do, Republic of Korea \\ Correspondence should be addressed to Jong-Lyel Roh; rohjl@amc.seoul.kr
}

Received 6 August 2017; Revised 19 October 2017; Accepted 23 October 2017; Published 31 December 2017

Academic Editor: Kum Kum Khanna

Copyright (C) 2017 Eun Hye Kim et al. This is an open access article distributed under the Creative Commons Attribution License, which permits unrestricted use, distribution, and reproduction in any medium, provided the original work is properly cited.

\begin{abstract}
Acquired resistance to cisplatin is the most common reason for the failure of cisplatin chemotherapy. Hederagenin, triterpenoids extracted from ivy leaves, exhibits antitumor activity in various types of cancer. However, the therapeutic potential of hederagenin in head and neck cancer (HNC) has remained unclear. Therefore, we examined the effects of hederagenin in cisplatin-resistant HNC cells and characterized its molecular mechanisms of action in this context. We evaluated the effects of hederagenin treatment on cell viability, apoptosis, reactive oxygen species (ROS) production, glutathione levels, mitochondrial membrane potential $(\Delta \Psi \mathrm{m})$, and protein and mRNA expression in HNC cells. The antitumor effect of hederagenin in mouse tumor xenograft models was also analyzed. Hederagenin selectively induced cell death in both cisplatin-sensitive and cisplatin-resistant HNC cells by promoting changes in $\Delta \Psi \mathrm{m}$ and inducing apoptosis. Hederagenin inhibited the Nrf2-antioxidant response element (ARE) pathway and activated p53 in HNC cells, thereby enhancing ROS production and promoting glutathione depletion. These effects were reversed by the antioxidant trolox. Hederagenin activated intrinsic apoptotic pathways via cleaved PARP, cleaved caspase-3, and Bax. The selective inhibitory effects of hederagenin were confirmed in cisplatinresistant HNC xenograft models. These data suggest that hederagenin induces cell death in resistant HNC cells via the Nrf2-ARE antioxidant pathway.
\end{abstract}

\section{Introduction}

Resistance to chemotherapy is a major obstacle to treating human cancer. Cisplatin is one of the most widely used chemotherapeutic agents in the treatment of various types of solid neoplasms [1]. Cisplatin is currently used as a firstline agent for the treatment of various cancers, including head and neck cancer (HNC), in combination with other anticancer chemotherapeutic agents and/or radiation therapy $[1,2]$. However, cisplatin is commonly associated with acquired resistance and increased toxicity, leading to poor tolerance and treatment outcomes $[3,4]$. HNC, the eighth most common cancer globally, typically manifests in the oral/nasal cavity, pharynx, and larynx of the upper aerodigestive tract $[5,6]$. A combined approach of surgery, radiotherapy, and chemotherapy is commonly used to treat HNC.
Nonsurgical chemoradiotherapy has increasingly been used as an organ-preserving treatment for patients with $\mathrm{HNC}$ $[7,8]$. Recent advances in cancer therapy have improved treatment outcomes; however, survival outcomes in patients with treatment-resistant HNC remain poor. Therefore, improving $\mathrm{HNC}$ treatment outcomes requires the development of novel approaches to treat chemotherapy-resistant cancers and identify more effective anticancer agents [7, 9].

Transcription factor nuclear factor (erythroid-derived 2)like 2 (Nrf2) plays a key role in regulating cellular redox homeostasis because its promoter binds to target genes containing the antioxidant response element (ARE) [10]. Cancer cells buffer cellular reactive oxygen species (ROS) levels by actively upregulating antioxidant pathways, including the Nrf2 pathway, that contribute to cancer therapy resistance $[11,12]$. Cellular metabolic pathways and antioxidant 
defense systems are commonly altered in treatment-resistant cancer cells exposed to high levels of oxidative stress [12, 13]. This metabolic alteration might represent a critical weakness that can be used as a basis to develop therapeutic approaches that selectively kill cancer cells and spare normal cells [14]. Therefore, antioxidant pathways and elevated ROS levels are increasingly gaining acceptance as promising targets in anticancer drug discovery [15].

Hederagenin is a triterpenoid isolated from ivy (Hedera helix L.) leaves [16], Chinese sweet tea (Cyclocarya paliurus) leaves [17], or other natural products [18]. Accumulating evidence indicates that hederagenin exerts significant cytotoxic effects in several types of cancers. Hederagenin saponin induced apoptosis in various types of human cancer cells by activating components of the mitochondria-mediated intrinsic apoptosis pathway, such as cleaved poly(ADP-ribose) polymerase (PARP), cleaved caspase-3, cleaved caspase-9, and $\mathrm{Bax}$, and by inhibiting the antiapoptotic protein $\mathrm{Bcl}-2$ [18]. In contrast, hederagenin saponin did not significantly affect proteins associated with the extrinsic cell death pathway, such as caspase-8 [16]. Hederagenin has been used as a triterpene template for the discovery of new anticancer compounds [19]. Although these findings suggest that the antitumor activity of hederagenin is mediated by the intrinsic cell death pathway, other mechanisms underlying hederagenin-mediated cancer cell death have yet to be elucidated. The antitumor effects of hederagenin have been examined in the lung, stomach, colon, and breast cancer cells but not in the HNC cells [16-18]. The in vitro and in vivo efficacy of hederagenin should be more examined in other types of human cancer, particularly those that exhibit resistance to current anticancer treatments. Therefore, we examined the effects of hederagenin in cisplatin-resistant HNC cells and characterized its molecular mechanism of action in this context. We found that hederagenin effectively induced apoptosis in cisplatin-resistant HNC cells in vitro and in vivo by targeting the Nrf2-ARE antioxidant pathway.

\section{Materials and Methods}

2.1. Cell Lines. We evaluated HNC cell lines (AMC-HN210), previously established at our institute, as well as SNU1041, SNU-1066, and SNU-1076 cell lines (Korea Cell Line Bank, Seoul, Republic of Korea). All of the cell lines were authenticated using short tandem repeat-based DNA fingerprinting and multiplex polymerase chain reaction (PCR). The cells were cultured in Eagle's minimum essential medium or Roswell Park Memorial Institute 1640 (Thermo Fisher Scientific, Waltham, MA, USA) supplemented with $10 \%$ fetal bovine serum. The cells were maintained at $37^{\circ} \mathrm{C}$ in a humidified atmosphere with $5 \% \mathrm{CO}_{2}$. Normal human oral keratinocytes and normal human fibroblasts (HOF) obtained from patients undergoing oral surgery were used for in vitro cell viability assays. The cisplatin-resistant HNC cell lines (HN3-cisR, HN4-cisR, and HN9-cisR) were generated by prolonged exposure of the cisplatin-sensitive parental cell lines ( HN3, HN4, and HN9 cells, resp.) to increase concentrations of cisplatin (Sigma-Aldrich, St. Louis, MO, USA). The half maximal inhibitory concentration $\left(\mathrm{IC}_{50}\right)$ of cisplatin, as determined using cell viability assays, was $2.2-3.5 \mu \mathrm{M}$ in the parental $\mathrm{HNC}$ cells and $25.5-38.9 \mu \mathrm{M}$ in the cisplatin-resistant HNC cells.

2.2. Cell Viability Assays. Cell viability following exposure to hederagenin (Biobank, Stockport, UK) was assessed using 3(4,5-dimethylthiazol-2-yl)-2,5-diphenyltetrazolium bromide (MTT) (Sigma-Aldrich), trypan blue exclusion, and clonogenic assays. Control cells were exposed to an equivalent amount of dimethyl sulfoxide (DMSO). Cell viability was also measured in hederagenin- or control-treated cells pretreated with trolox (Enzo Life Sciences Inc., Farmingdale, NY, USA), trigonelline (Sigma-Aldrich), or MG132 (SigmaAldrich). MTT assays were performed by incubating the cells with the tetrazolium compound for $4 \mathrm{~h}$ followed by solubilization buffer for $2 \mathrm{~h}$. The absorbance at $570 \mathrm{~nm}$ was subsequently measured using a SpectraMax M2 microplate reader (Molecular Devices, Sunnyvale, CA, USA). Trypan blue exclusion assays were performed by staining the cells with $0.4 \%$ trypan blue and counting the stained cells using a hemocytometer. Clonogenic assays were performed by incubating the cells with $0.5 \%$ crystal violet solution and counting the number of colonies ( $>50$ cells) after 14 days of culturing.

Cell death was analyzed by staining the cells with annexin $\mathrm{V}$ and propidium iodide (PI) (Sigma-Aldrich). Annexin Vand PI-positive cells were quantitatively analyzed using flow cytometry and Cell Quest Pro software (BD Biosciences, Franklin Lakes, NJ, USA). To measure the mitochondrial membrane potential $(\Delta \Psi \mathrm{m})$, the cells were stained with $200 \mathrm{nM}$ tetramethylrhodamine ethyl ester (TMRE) (Thermo Fisher Scientific) for 20 min and analyzed using flow cytometry. The median fluorescence intensity (MFI) of each treatment group was normalized to that of the control group. All assays were performed in triplicate using three samples in each assay.

2.3. Measuring Glutathione (GSH) Synthesis and ROS Production. Cellular GSH levels were measured in HNC cell lysates using a GSH colorimetric detection kit (BioVision Inc., Milpitas, CA, USA). $2^{\prime}, 7^{\prime}$-Dichlorofluorescein diacetate (DCF-DA) (Enzo Life Sciences, Farmingdale, NY, USA) was used to measure cellular ROS levels in HNC cell lysate supernatants. ROS levels were analyzed using a FACSCalibur flow cytometer equipped with CellQuest Pro software (BD Biosciences).

2.4. RNA Interference and Gene Transfection. To silence SQSTM1(p62) and NFE2L2 (Nrf2) expression, cisplatinresistant HN4-cisR cells were seeded and transfected $24 \mathrm{~h}$ later with $10 \mathrm{nmol} / \mathrm{L}$ small interfering RNA (siRNA) targeting human NFE2L2 or KEAP1 or with a scrambled control siRNA (Integrated DNA Technologies, Coralville, IA, USA). siRNA-induced gene silencing was confirmed using reverse transcription-quantitative PCR (RT-qPCR) analysis of $1-2 \mu \mathrm{g}$ of total RNA from each sample with a SuperScript ${ }^{\circledR}$ III RT-PCR system (Thermo Fisher Scientific) and Western blot assays with anti-p62 and anti-Nrf2 
antibodies. To generate cells stably overexpressing Nrf2, HN3 cells were stably transfected with a control plasmid or an Nrf2-expressing plasmid (Transomic, Huntsville, AL). Nrf2 overexpression was confirmed using RT-qPCR and Western blotting.

2.5. Western Blot Assays. The cells were plated, grown to $70 \%$ confluence, and subsequently treated with the indicated reagents. The cells were lysed at $4^{\circ} \mathrm{C}$ in radioimmunoprecipitation assay (RIPA) lysis buffer (Thermo Fisher Scientific). A total of $50 \mu \mathrm{g}$ of protein were resolved using SDS-PAGE on $10 \%-12 \%$ gels, and the separated proteins were transferred to nitrocellulose or polyvinylidene difluoride membranes. The membranes were probed with primary and secondary antibodies. Primary antibodies against the following proteins were used: poly(ADP-ribose) polymerase (PARP) and cleaved PARP, cleaved caspase-3, p53, phospho-p53Ser15, p62, Bax, Bcl-2, Nrf2, heme oxygenase-1 (HO-1), $\mathrm{NAD}(\mathrm{P}) \mathrm{H}$ : quinone oxidoreductase 1 (NQO1) (Cell Signaling Technology, Danvers, MA), xCT (Abcam, Cambridge, UK), and Keap1 (Santa Cruz Biotechnology, Santa Cruz, CA, USA). $\beta$-Actin (Sigma-Aldrich) was used as a loading control. All of the antibodies were used at a dilution of $1: 250-1: 5000$.

2.6. Nrf2 Transcriptional Activity. The Nrf2 transcriptional activity was assayed using a Cignal Antioxidant Response Reporter kit (Qiagen, Valencia, CA, USA) according to the manufacturer's instructions.

2.7. Immunofluorescence Staining. The cells were incubated with antibodies against p62 and Nrf2. 4',6-Diamidino-2-phenylindole (DAPI) (Thermo Fisher Scientific) was used as a counterstain to label cell nuclei. The cells were fixed using 3.7\% paraformaldehyde in prewarmed complete medium for $15 \mathrm{~min}$ at $37^{\circ} \mathrm{C}$. The fixed cells were deparaffinized, rehydrated, and incubated with the indicated primary and secondary antibodies. The stained cells were observed and imaged using a fluorescent microscope. Mitochondrial superoxide generation in live hederagenin-treated cells was quantitatively analyzed using mitoSOX (Thermo Fisher Scientific). The stained cells were observed using a fluorescent microscope. The mean fluorescence intensity in each group was normalized to that in the control group.

2.8. Tumor Xenografts. All of the animal procedures were performed in accordance with protocols approved by the Institutional Animal Care and Use Committee of our institution. Six-week-old athymic BALB/c male nude mice (nu/nu) were purchased from Central Lab Animal Inc. (Seoul, Republic of Korea). HN9-cisR cells were subcutaneously injected into the flank of nude mice. Beginning on the first day gross nodules from tumor implants were detected, the mice began receiving intraperitoneal injections of the vehicle control or hederagenin $(50,100$, or $200 \mathrm{mg} / \mathrm{kg}$ daily). Each treatment group included 10 mice. The tumor size and body weight were measured twice a week, and the tumor volume was calculated as length $\times$ width $^{2} / 2$. The mice were sacrificed on day 35 , and the tumors were isolated and analyzed for cellular GSH levels. Apoptosis in tumors was analyzed using an in situ terminal deoxynucleotidyl transferase-mediated dUTP nick-end labeling (TUNEL) assay (Promega, Fitchburg, WI, USA), and the number of apoptotic bodies in 10 randomly selected high-power fields was counted in a blinded manner.

2.9. Statistical Analysis. The data are presented as the mean \pm standard error of the mean. The statistical significance of differences between treatment groups was assessed using the Mann-Whitney $U$ test or analysis of variance with Bonferroni post hoc test. The data were analyzed using SPSS version 23.0 (IBM, Armonk, NY, USA). Statistical significance was defined as a two-sided $P$ value $<0.05$.

\section{Results and Discussion}

3.1. Hederagenin Induces Apoptosis in Cisplatin-Sensitive and Cisplatin-Resistant HNC Cells. The molecular weight of hederagenin is $472.7 \mathrm{~g} / \mathrm{mol}$ (Figure 1(a)). Hederagenin decreased the viability of cisplatin-sensitive and cisplatinresistant cancer cells in a dose-dependent manner (Figures 1(b) and 1(c)). The viability decreased by up to $50 \%$ in cells treated with $20 \mu \mathrm{M}$ hederagenin and by up to $90 \%$ in cells treated with $80 \mu \mathrm{M}$ hederagenin for $72 \mathrm{~h}$. Cisplatin-resistant HNC cells were less sensitive to hederagenin treatment compared with cisplatin-sensitive HNC cells. However, the cell viability was nearly abolished in all the cisplatin-sensitive and cisplatin-resistant HNC cells treated with hederagenin concentrations $>80 \mu \mathrm{M}$. Representative images of hederagenin-treated cells are presented in Figure 1(d).

Hederagenin inhibited the growth of cisplatin-resistant HNC cells in a treatment time- and dose-dependent manners (Figure 2(a)), and pretreatment with the antioxidant trolox $(0.5 \mathrm{mM})$ inhibited this effect. In addition, hederagenin significantly suppressed colony formation in cisplatin-resistant HNC cells, and this effect was also significantly inhibited by trolox pretreatment $(P<0.05)$ (Figure $2(b))$. Hederagenin induced apoptotic cell death in all cisplatin-resistant HNC cell lines evaluated. This effect was observed as early as $24 \mathrm{~h}$ after treatment, and it increased in a time-dependent treatment manner (Figures 2(c) and 2(d)).

Previous studies demonstrated that hederagenin induced cell death in human cancer cells by activating intrinsic apoptotic pathways [16-18]. A hederagenin saponin, macranthoside $B$, extracted from the Chinese plant Lonicera macranthoides, exerted cytotoxic effects in cancer cells. It also exhibited anti-inflammatory activity and provided protection against liver damage $[18,20]$. The strong antitumor effect of hederagenin saponins is mediated by the activation of mitochondria-mediated apoptosis in hepatocellular carcinoma cells [18]. Hederagenin also strongly upregulates the apoptotic protein Bax and downregulates the antiapoptotic protein $\mathrm{Bcl}-2$, thereby increasing the $\mathrm{Bax} / \mathrm{Bcl}-2$ ratio in cancer cells [18]. Hederagenin isolated from ivy leaves (Hedera helix L.) also induced apoptosis via the mitochondrial pathway in colon cancer cells [16]. Hederagenin activates the apoptosis executioner caspases, caspase-3, caspase-6, and caspase-9, thereby promoting 


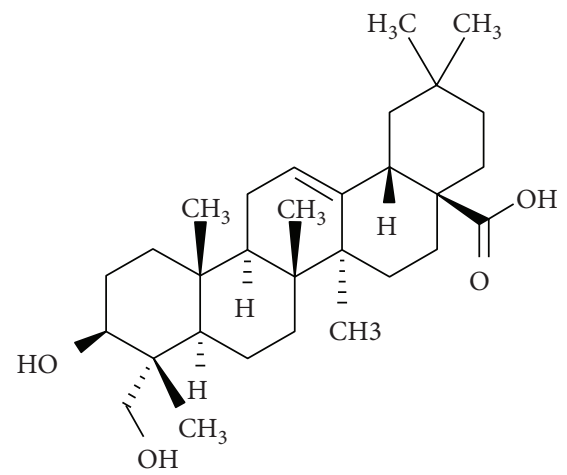

Hederagenin

(a)

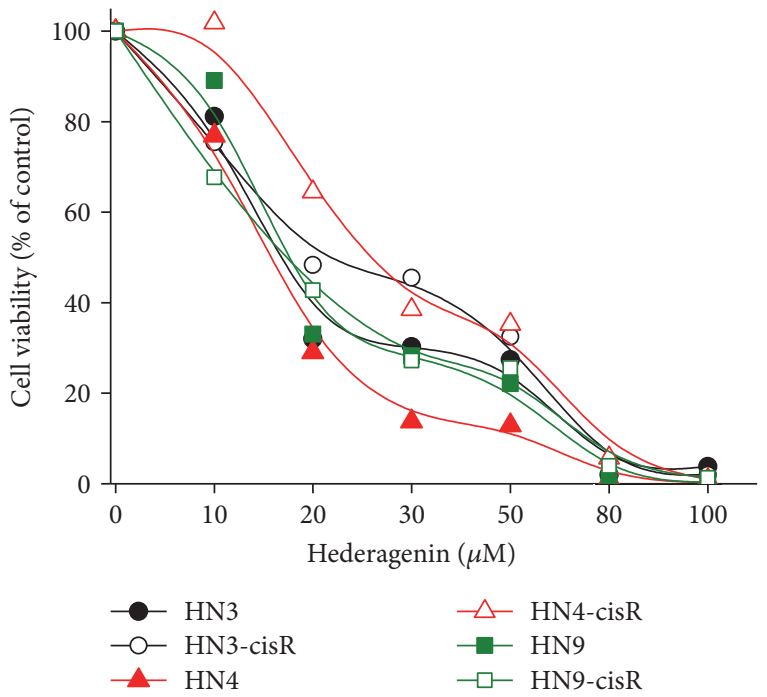

(c)

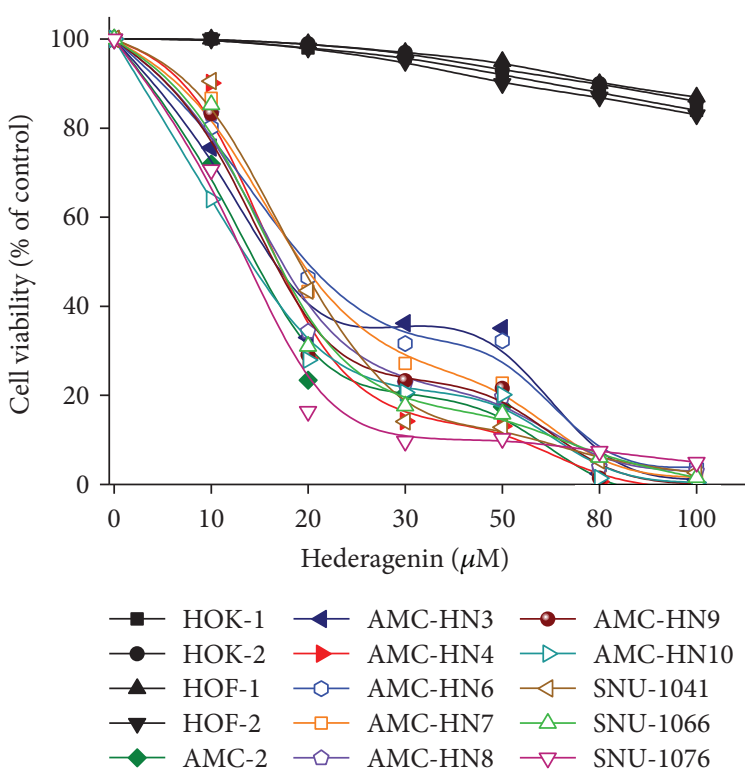

(b)
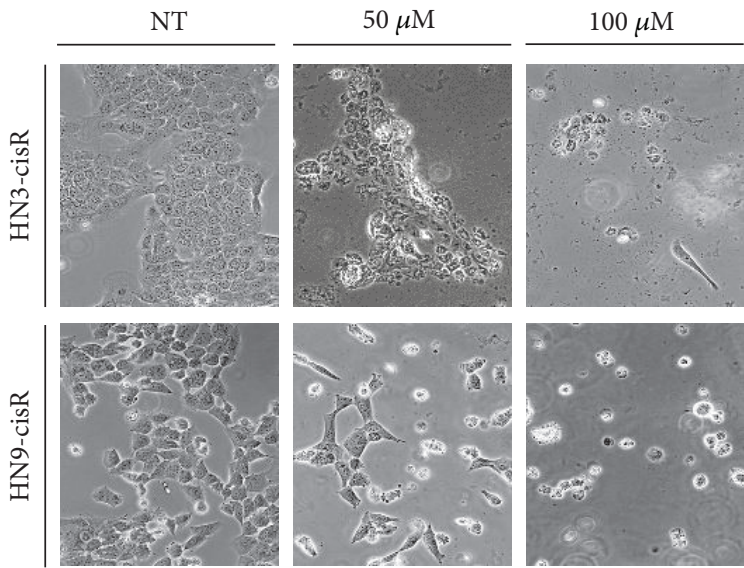

Figure 1: Hederagenin decreases the viability of HNC cells. (a) The structure of hederagenin. (b, c) Cell viability was assessed in cisplatin-sensitive and cisplatin-resistant $\mathrm{HNC}$ cell lines exposed to various concentrations of hederagenin for $72 \mathrm{~h}$. (d) Images of hederagenin-treated and hederagenin-nontreated (NT) cisplatin-resistant HNC cells.

cytochrome $c$ release, but it does not activate caspase- 8 , a protein associated with the extrinsic apoptosis pathway $[16,21]$. Hederagenin extracted from the leaves of the Chinese sweet tea Cyclocarya paliurus also selectively exerted cytotoxic effects in breast and lung cancer cells [17]. Hederagenin induces mitochondria-driven apoptosis and anti-inflammatory effects by suppressing the NF- $\kappa \mathrm{B}$ pathway, similar to the action of NF- $\kappa$ B inhibitors [17]. Consistent with the previous findings, the present study demonstrated that the prooxidant effect of hederagenin selectively induced apoptosis in cisplatin-resistant cancer cells while sparing normal cells.
3.2. Hederagenin Induces Cellular GSH Depletion and ROS Accumulation in HNC Cells. Cellular GSH levels significantly decreased and cellular ROS levels significantly increased in hederagenin-treated cells $(P<0.05)$ (Figures $3(\mathrm{a})$ and $3(\mathrm{~b}))$, and these effects were significantly inhibited by pretreatment with $0.5 \mathrm{mM}$ trolox $(P<0.05)$. Cisplatin treatment alone did not affect the cellular levels of GSH and ROS. In addition, hederagenin induced changes in $\Delta \Psi \mathrm{m}$ in cisplatin-resistant HNC cells, as demonstrated by a decrease in TMRM staining and an increase in mitoSOX staining (Figures 3(c) and 3(d)). This effect was reversed by trolox pretreatment, and cisplatin alone did not affect $\Delta \Psi \mathrm{m}$. 


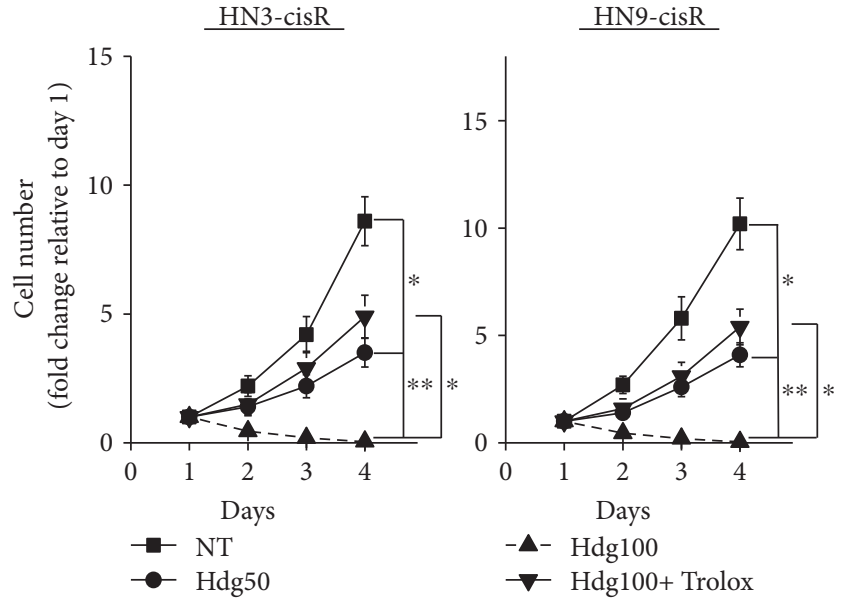

(a)

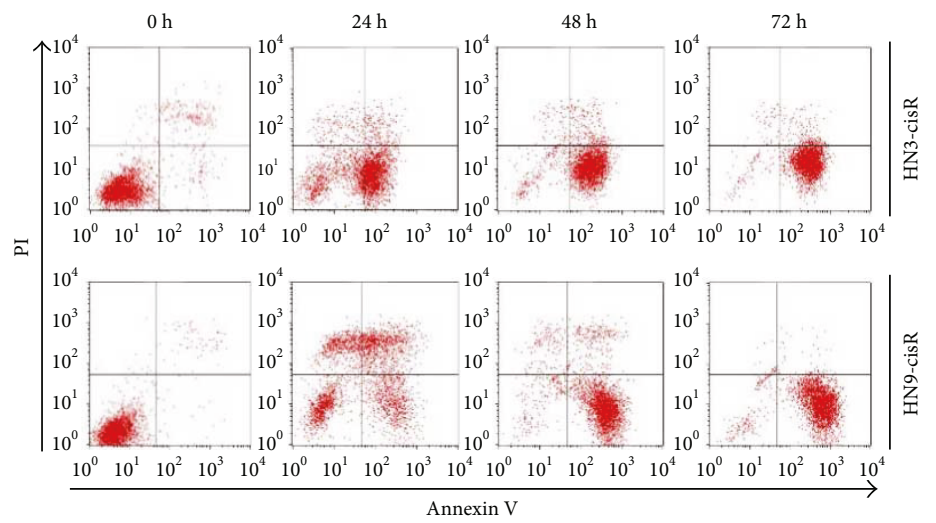

(c)

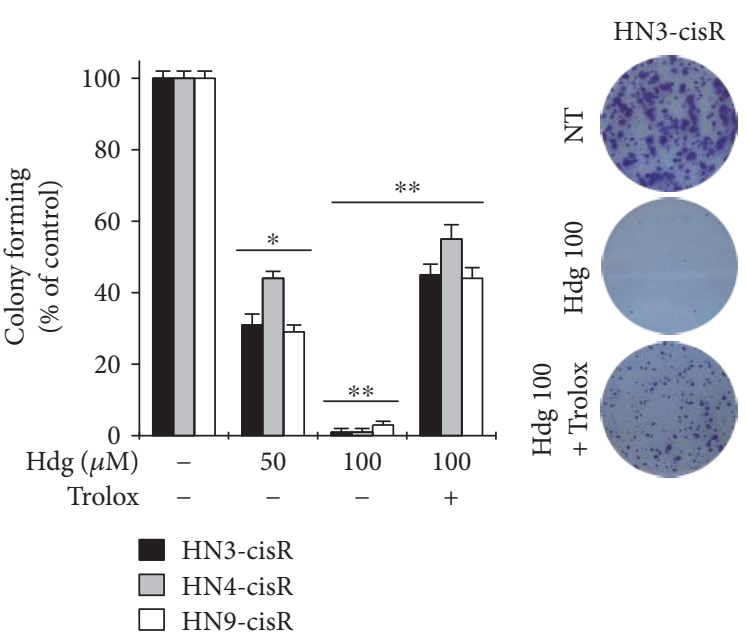

(b)

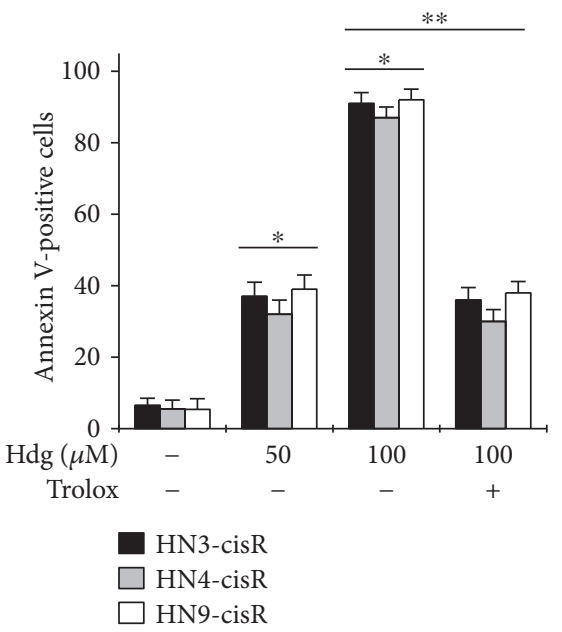

(d)

Figure 2: Hederagenin induces apoptotic cell death in cisplatin-resistant HNC cells. (a, b) Changes in cell number and colony-forming ability in cisplatin-resistant HNC cells exposed to hederagenin (Hdg) with or without pretreatment with the antioxidant trolox (0.5 mM). NT: control cells not treated with hederagenin. (c) FACS analyses of Annexin V and propidium iodide staining of cisplatin-resistant HNC cells treated with $100 \mu \mathrm{M}$ hederagenin. (d) Apoptosis in cells treated with various concentrations of hederagenin for $72 \mathrm{~h}$ was assessed by quantifying the proportion of Annexin V-positive cells. The error bars represent the standard error from three independent experiments. ${ }^{*} P<0.05,{ }^{* *} P<0.01$ relative to the control or between groups.

Our study focused on the effect of hederagenin on the modulation of cellular oxidation. Hederagenin enhanced ROS production in HNC cells by promoting GSH depletion, and this effect was reversed by the antioxidant trolox. Consistent with the previous findings, the present study demonstrated that hederagenin induces apoptosis in cancer cells by downregulating $\Delta \Psi \mathrm{m}$. We also demonstrated that hederagenin activates regulator upstream of the intrinsic apoptotic pathway in HNC cells.

3.3. Hederagenin Inhibits the Nrf2-ARE Pathway. Hederagenin enhanced the levels of proapoptotic proteins (cleaved PARP, cleaved caspase-3, and BAX), whereas it suppressed the levels of the antiapoptotic protein Bcl-2. Hederagenin enhanced p53 levels in HN9 and HN9-cisR cells, which express wild-type p53 (Figure 4(a)), whereas it did not significantly affect p53 levels in HN3 or HN3-cisR cells, which express mutant p53 (R282W). However, phospho-p53 levels increased in hederagenin-treated HN3 and HN3-cisR cells. In addition, levels of the Nrf2-ARE antioxidant pathway components Nrf2, HO-1, NQO1, and xCT decreased in cisplatin-resistant $\mathrm{HNC}$ cells treated with hederagenin (Figure 4(b)), whereas Nrf2 mRNA expression was not significantly affected $(P>0.1)$ (Figure $4(c))$. The level of Keap1 increased in the HNC cells by hederagenin treatment along with the decreased level of p62. Regardless of treatment in combination with the proteasome inhibitor MG132, hederagenin decreased the levels of Nrf2, xCT, and p62 in a time- 


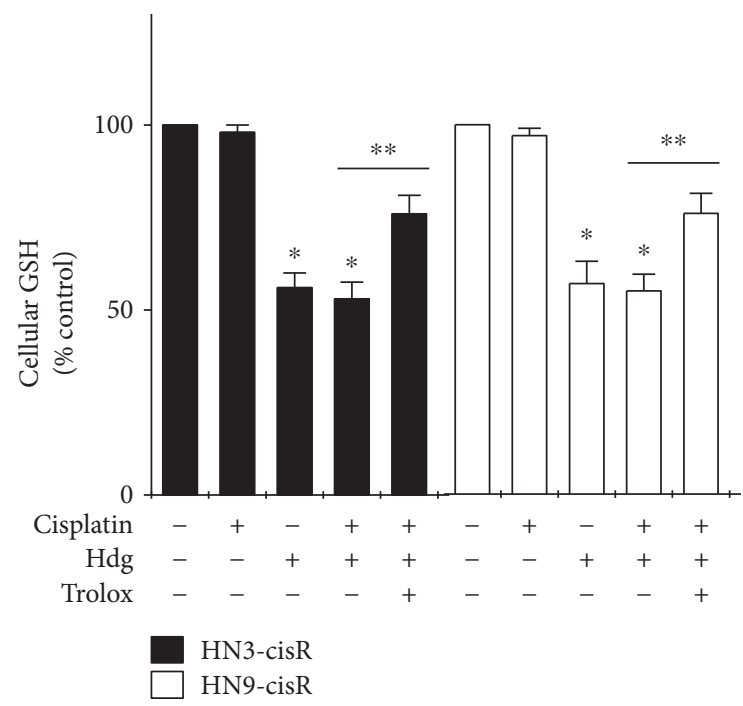

(a)

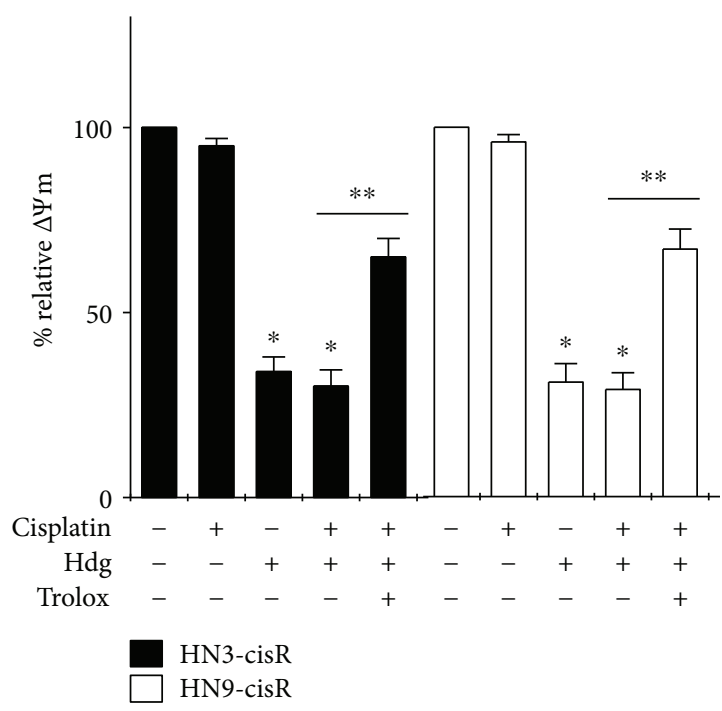

(c)

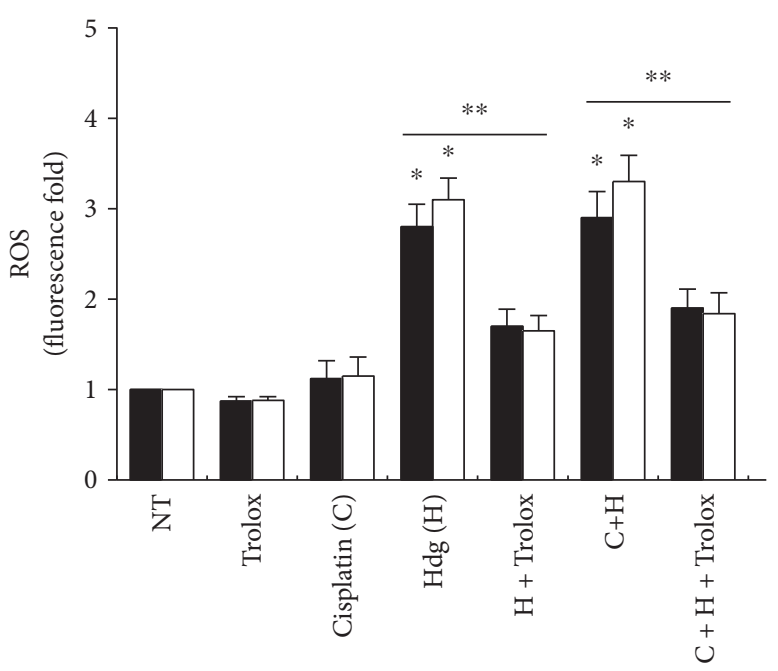

HN3-cisR

HN9-cisR

(b)
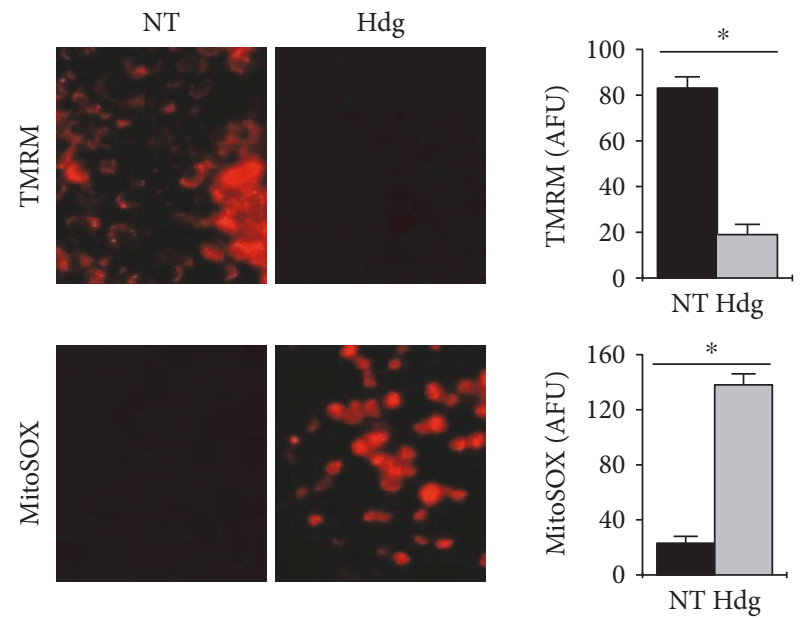

FIGURE 3: Hederagenin induces GSH depletion and ROS accumulation in cisplatin-resistant HNC cells. (a, b) Cellular GSH and ROS levels in cells treated with various combinations of $10 \mu \mathrm{M}$ cisplatin, $80 \mu \mathrm{M}$ hederagenin, and $0.5 \mathrm{mM}$ trolox for $24 \mathrm{~h}$. (c, d) Changes in the mitochondrial membrane potential $(\Delta \Psi \mathrm{m})$ in cisplatin-resistant HNC cells treated with various combinations of $10 \mu \mathrm{M}$ cisplatin, $80 \mu \mathrm{M}$ hederagenin, and $0.5 \mathrm{mM}$ trolox for $24 \mathrm{~h}$. $\Delta \Psi \mathrm{m}$ was measured using flow cytometry analysis of cells stained with TMRE. The MFI of each treatment group was normalized to the control group. The error bars represent the standard error from three independent experiments. ${ }^{*} P<0.05$ relative to control, ${ }^{* *} P<0.05$ between groups.

dependent manner (Figure 4(d)). Hederagenin-induced changes in Nrf2 and p62 were confirmed by the results of immunofluorescence staining assays (Figure 4(e)). Immunoblotting analysis of the cytoplasmic and nuclear extracts of HNC cells demonstrated that changes in Nrf2 levels in cisplatin-resistant HNC cells treated with hederagenin primarily occurred in the cytoplasm (Figure 4(f)). Hederagenin also inhibited the Nrf2 transcriptional activity and $\mathrm{HO}-1$ and NQO1 mRNA levels in HNC cells (Figures 4(g) and 4(h)).
Nrf2 overexpression suppressed the inhibitory effects of hederagenin on cell growth, but it did not accelerate the growth of HNC cells in the absence of hederagenin (Figures 5(a) and 5(b)). Nrf2 overexpression resulting from siRNA-mediated Keap1 knockdown also suppressed the inhibitory effects of hederagenin on HNC cell growth (Figures 5(c) and 5(d)). However, neither siRNAmediated Nrf2 knockdown nor trigonelline-mediated pharmacological inhibition of $\mathrm{Nrf} 2$ enhanced the effects of 


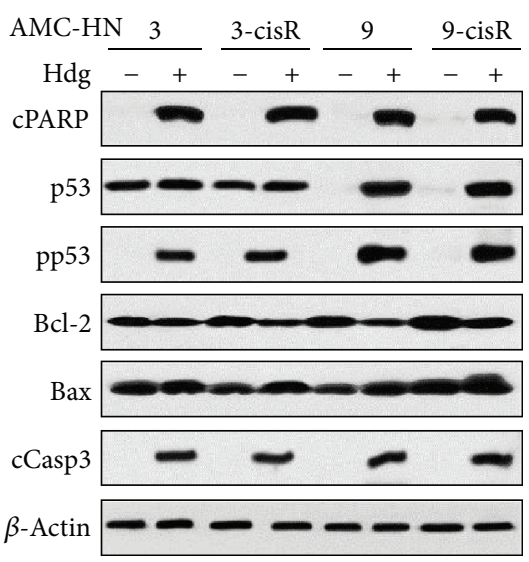

(a)

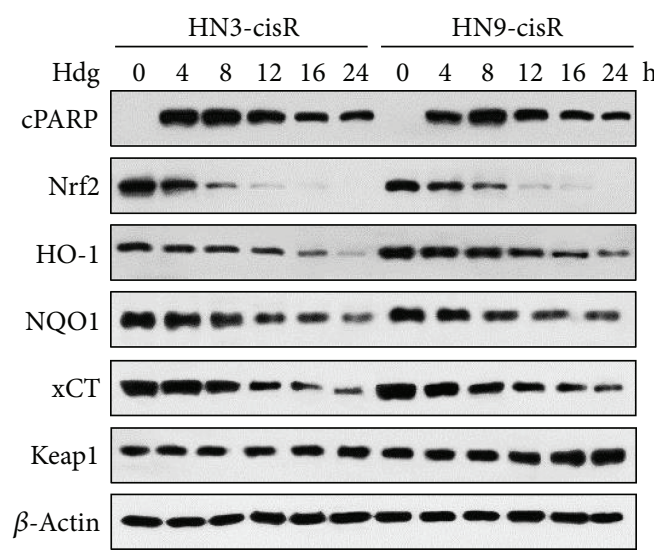

(b)

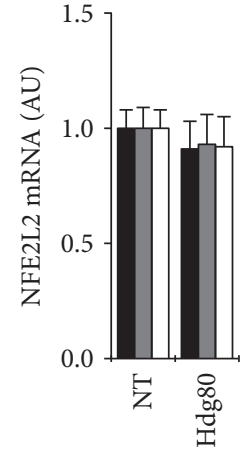

HN3-cisR

$\square$ HN4-cisR

$\square$ HN9-cisR

(c)

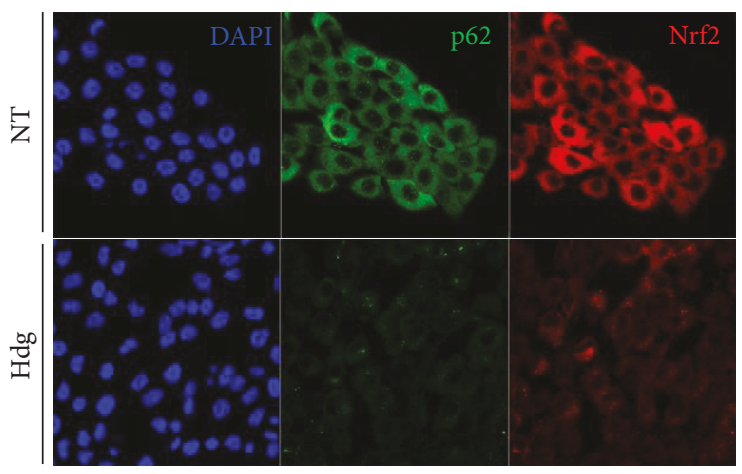

(e)

(d)

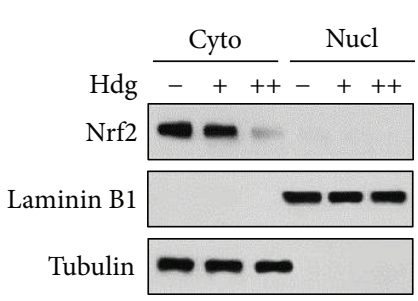

(f)

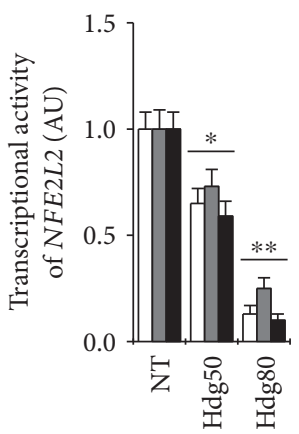

HN3-cisR $\square$ HN4-cisR HN9-cisR

(g)
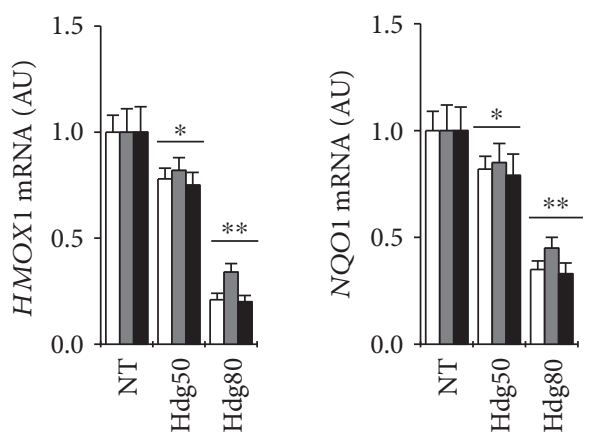

(HN3-cisR

$\square$ HN4-cisR

$\square$ HN9-cisR 

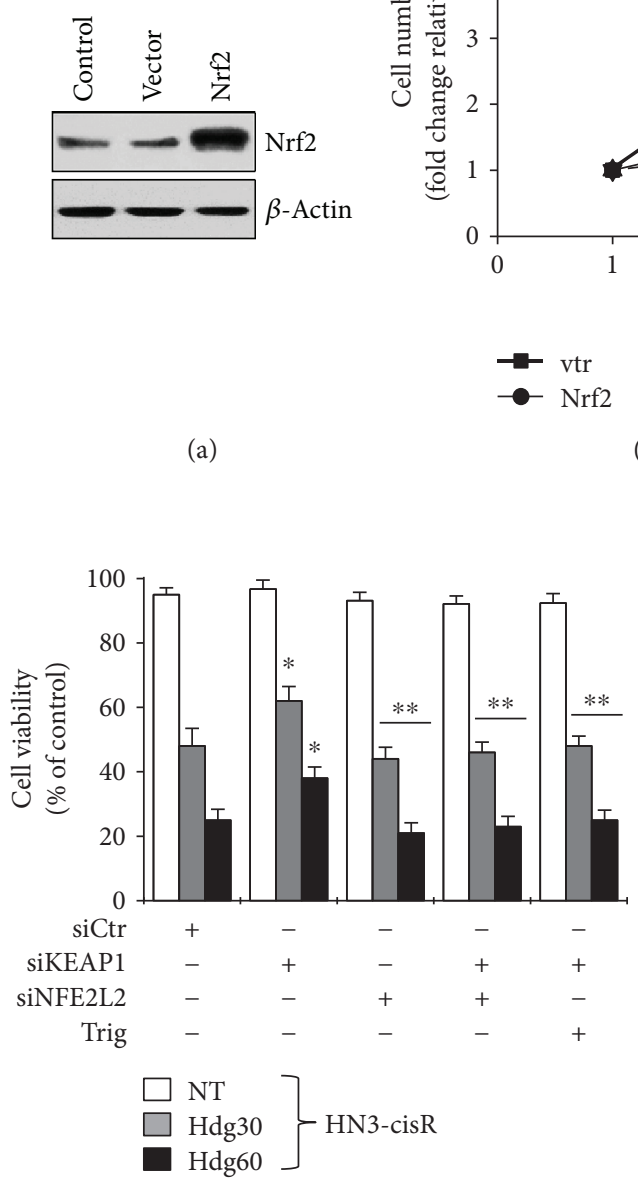

(d)

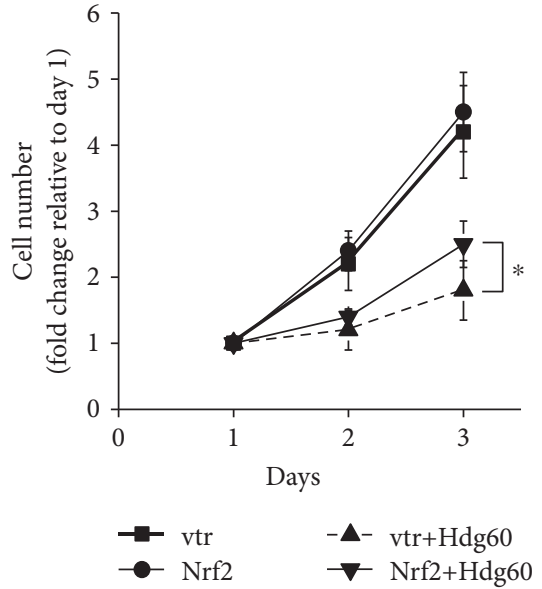

(b)
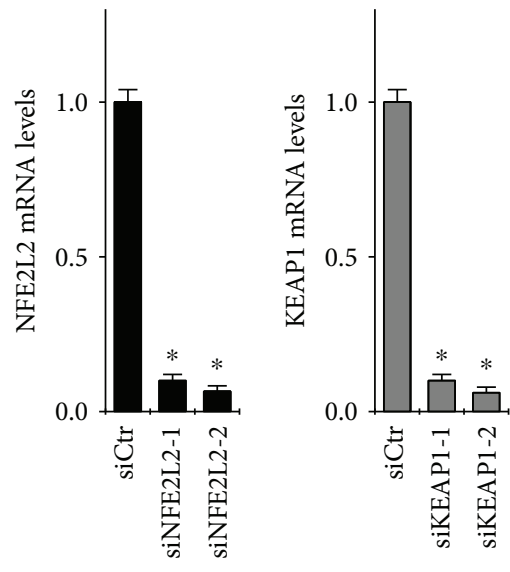

(c)
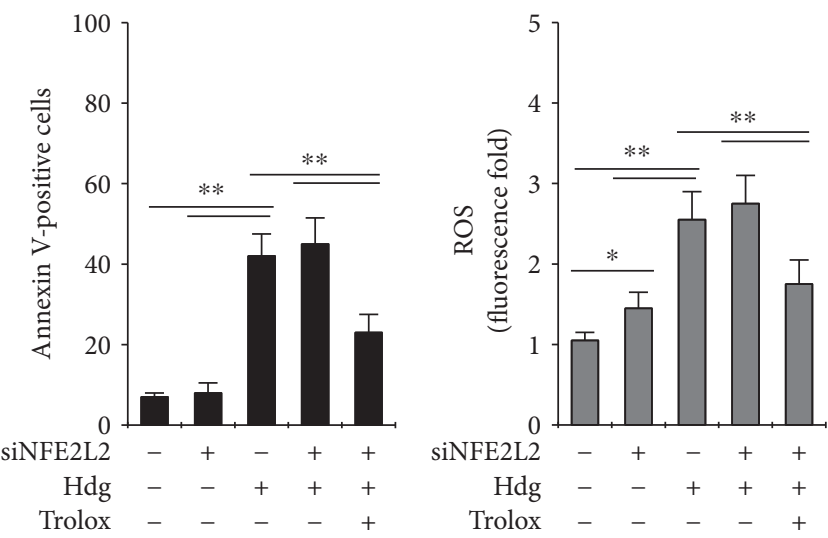

(f)

FIGURE 5: Hederagenin induces cell death in HNC cells by inhibiting Nrf2. (a, b) Effects of Nrf2 overexpression on hederagenin-induced changes in cell growth. (c, d) Cell viability in HN3-cisR cells transfected with siControl (siCtr), siNFE2L2, or siKEAP1 and exposed to hederagenin in the presence or absence of trigonelline (Trig, $100 \mu \mathrm{M}$ ). ${ }^{*} P<0.05$ relative to the siCtr; ${ }^{* *} P<0.05$ relative to the siKEAP1 group with $30 \mu \mathrm{M}$ or $60 \mu \mathrm{M}$ hederagenin treatment. (e, f) FACS analysis and ROS levels in HN3-cisR cells transfected with siCtr or siNFE2L2 and treated with $60 \mu \mathrm{M}$ hederagenin, $0.5 \mathrm{mM}$ trolox, or both. Error bars represent the standard error from three replicate experiments. ${ }^{*} P<0.05,{ }^{* *} P<0.01$ relative to the control or between groups.

hederagenin on cell viability, apoptosis, or cellular ROS accumulation (Figures 5(d), 5(e), and 5(f)). The effects of hederagenin on apoptosis and ROS accumulation were significantly inhibited in cells pretreated with trolox.

The current study demonstrated that hederagenin selectively induces HNC cell death by enhancing ROS production and promoting the depletion of GSH via inhibition of the Nrf2-ARE pathway. Nrf2 plays a central role in the cellular response to oxidative damage [22]. It regulates the expression of target genes associated with the cellular antioxidant systems that promote GSH production [14]. Nrf2 is constantly degraded by the proteosomal activity of Keap1; therefore, Nrf2 activity is upregulated by Keap1 inhibition [22, 23]. A growing body of evidence indicates that the Keap1-Nrf2 system plays an important role in carcinogenesis and chemotherapy resistance [24-27]. We previously demonstrated that Nrf2 plays a role in cisplatin resistance in HNC [28]. The present study demonstrated that cytoplasmic levels of $\mathrm{Nrf} 2$ increased in cisplatin-resistant HNC cells, consistent with its proposed association with tumor aggressiveness [29], and this effect was strongly inhibited by hederagenin treatment. In addition, we demonstrated that hederagenin upregulated wild-type p53 and phospho-p53 in HNC cells, an effect that partially suppressed Nrf2-dependent transcription of antioxidant response genes and activated proapoptosis proteins [30]. The effects of hederagenin on Nrf2 were observed in all of the tumor cells evaluated, regardless of the P53 mutation status. As P53 mutations can contribute to treatment resistance, this observation might partially account for the 


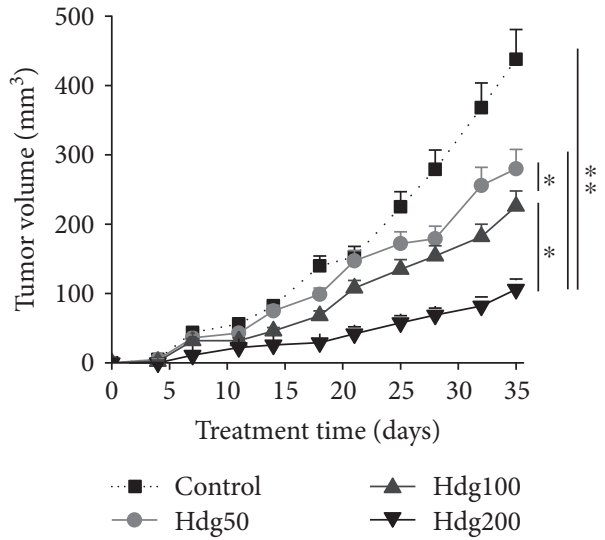

(a)

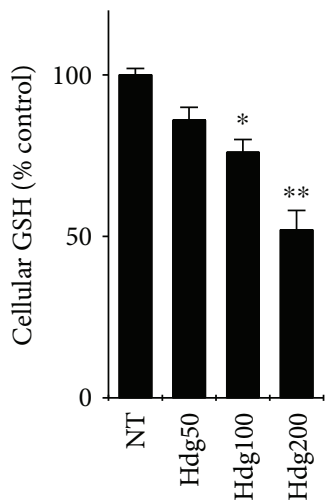

(d)
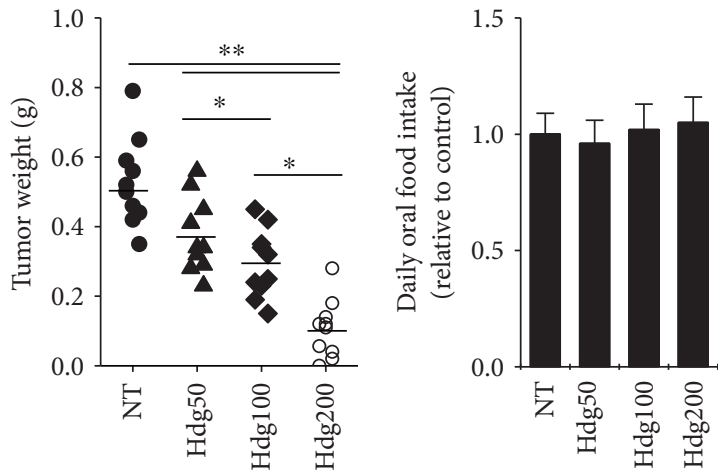

(b)

(c)
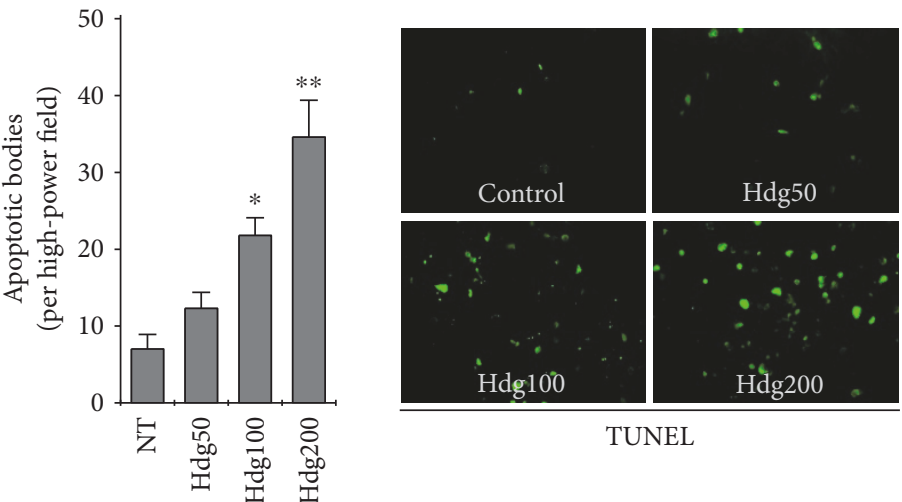

TUNEL

(e)

FIGURE 6: Hederagenin inhibits the growth of cisplatin-resistant HNC cell tumor xenografts. (a, b) Growth and weight of HN9-cisR tumors in nude mice. The mice received intraperitoneal injections of the vehicle control (NT) or 50, 100, or $200 \mathrm{mg} / \mathrm{kg}$ hederagenin. (c) Comparison of daily food intake among different treatment groups. (d) Cellular GSH levels in tumors treated with vehicle or hederagenin. (e) TUNELpositive apoptotic bodies in 10 randomly selected high-power fields were counted in a blinded manner. The error bars represent standard errors. ${ }^{*} P<0.05,{ }^{* *} P<0.01$ relative to the control or other treatment groups.

ability of hederagenin to effectively target cisplatin-resistant HNC cells.

Both siRNA-mediated Nrf2 knockdown and pharmacological inhibition of Nrf2 markedly suppress GSH levels and enhance the cytotoxic effects of chemotherapeutic agents $[26,27]$. Cancer cells are characterized by high levels of oxidative stress due to elevated ROS levels, and this defect alters various cellular metabolic pathways and activates antioxidant defense mechanisms [12, 13]. Therefore, oxidative stress might represent a critical weakness that can be targeted by selective therapeutic approaches to various types of cancer [14]. Combinations of antioxidant inhibitors and chemotherapeutic agents promote oxidative stress in cancer cells, thereby selectively promoting cell death in cancer cells $[31,32]$. Therefore, elevated ROS levels are increasingly accepted as a valuable therapeutic target in anticancer drug discovery $[14,15]$. We previously demonstrated that wogonin, a natural active flavonoid, inhibited Nrf2 upregulation in cisplatin-resistant HNC cells [28], similar to what has been observed with other anticancer drugs [26,33]. In the present study, hederagenin inhibited the Nrf2-ARE pathway in cisplatin-resistant HNC cells, thereby inducing cytotoxic effects. Collectively, our results suggest that the
Nrf2 pathway is a potential target of future therapies for chemoresistant HNC.

\subsection{Hederagenin Inhibits the Growth of Cisplatin-Resistant} HNC In Vivo. In mouse xenograft models injected with HN9-cisR cells, hederagenin significantly suppressed tumor growth in vivo in a dose-dependent manner (Figures 6(a) and 6(b)). Hederagenin did not significantly affect daily food intake or body weight compared with the vehicle control (Figure 6(c)). Hederagenin significantly suppressed GSH levels in tumor cells compared with the vehicle control $(P<0.05)$ (Figure $6(\mathrm{~d}))$. In addition, the number of TUNELpositive apoptotic bodies in tumors significantly increased in the hederagenin-treated group compared with that in the control group $(P<0.05)$ (Figure $6(\mathrm{e}))$. At the histological level, there were no significant differences in vital organs between the treatment groups (data not shown).

Hederagenin has been reported to exert a cytoprotective effect on normal tissues $[34,35]$. A recent study demonstrated that hederagenin might prevent alcoholic liver injury via its anti-inflammatory and antiapoptotic activities [34]. The expression of apoptotic proteins and proinflammatory cytokines is lower in normal cells than in cancer 


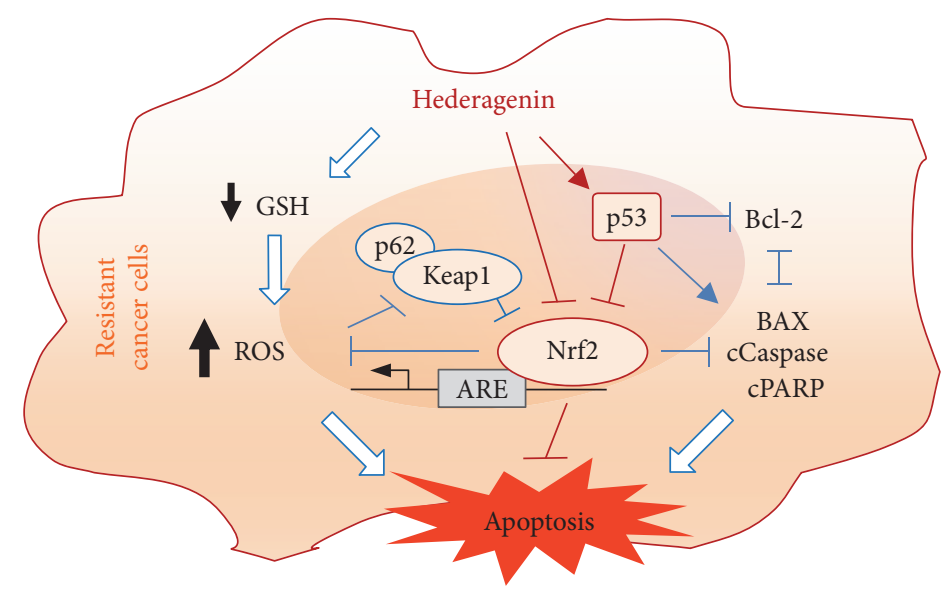

FIGURE 7: A model of the proposed mechanism of hederagenin in cisplatin-resistant HNC cells. Hederagenin selectively induces apoptosis in cancer cells by promoting ROS production and GSH depletion via inhibition of the Nrf2-ARE pathway.

cells. Hederagenin demonstrated therapeutic effects in neurodegenerative diseases such as Parkinson's disease and Huntington's disease by inducing autophagy and promoting the degradation of disease-associated proteins [35]. In the present study, hederagenin selectively induced apoptosis in cancer cells while sparing normal cells. Interestingly, hederagenin treatment was not associated with weight loss or histological changes in major organ systems. As evidence demonstrating the anticancer activity of hederagenin has been accumulated, novel hederagenin derivatives are being developed for use as potential anticancer agents [19]. Compared with the parental molecule, hederagenin derivatives appear to have a more potent effect on apoptosis induction in human cancer cell lines [19]. Therefore, hederagenin is more likely to be used as a triterpene template for the discovery of new anticancer compounds.

\section{Conclusion}

The present study revealed a novel mechanism by which hederagenin enhances ROS levels in cisplatin-resistant cancer cells by inhibiting the Nrf2-ARE pathway, a central player in redox homeostasis (Figure 7). Our findings suggest that hederagenin effectively targets cisplatin-resistant HNC cells in vitro and in vivo. Consistent with its effects in other types of cancer, hederagenin markedly induces apoptosis in HNC cells by activating the mitochondria-driven intrinsic apoptotic pathway. We demonstrated that the apoptosis-inducing effects of hederagenin are mediated by the inhibition of the Nrf2-ARE antioxidant pathway. Additional preclinical and clinical investigations of this promising anticancer therapy in patients with other types of treatment-resistant cancer are warranted.

\section{Abbreviations}
ARE:
Antioxidant response element
DCF-DA:
$2^{\prime}, 7^{\prime}$-Dichlorofluorescein diacetate
GSH:
Glutathione
HNC:

HO-1: $\quad$ Heme oxygenase-1

Keap1: $\quad$ Kelch-like ECH-associated protein 1

MFI: $\quad$ Median fluorescence intensity

$\triangle \Psi \mathrm{m}: \quad$ Mitochondrial membrane potential

NQO1, NAD(P)H: Quinone oxidoreductase 1

Nrf2: Nuclear factor (erythroid-derived 2)like 2

PARP: $\quad$ Poly(ADP-ribose) polymerase

PI: $\quad$ Propidium iodide

ROS: $\quad$ Reactive oxygen species

RT-qPCR: Reverse transcription-quantitative polymerase chain reaction

siRNA: $\quad$ Short interfering RNA

TMRE: Tetramethylrhodamine ethyl ester

TUNEL: Terminal deoxynucleotidyl transferase dUTP nick end labeling

xCT: Cystine/glutamate antiporter system $\mathrm{xc}^{-}$.

\section{Conflicts of Interest}

The authors declare no conflict of interest.

\section{Authors' Contributions}

Eun Hye Kim and Seungho Baek contributed equally to this work.

\section{Acknowledgments}

This study was supported by a grant (no. 2015R1A2A1A15054540) from the Basic Science Research Program through the National Research Foundation of Korea (NRF), Ministry of Science, ICT, and Future Planning, Seoul, Republic of Korea (Jong-Lyel Roh), and a grant (NRF2017R1C1B1002724) from the National Research Foundation of Korea (NRF), Ministry of Science, ICT, and Future Planning, Seoul, Republic of Korea (Seungho Baek). 


\section{References}

[1] L. Galluzzi, I. Vitale, J. Michels et al., "Systems biology of cisplatin resistance: past, present and future," Cell Death and Disease, vol. 5, no. 5, article e1257, 2014.

[2] R. I. Haddad and D. M. Shin, "Recent advances in head and neck cancer," The New England Journal of Medicine, vol. 359, no. 11, pp. 1143-1154, 2008.

[3] F. Petrelli, A. Coinu, V. Riboldi et al., "Concomitant platinumbased chemotherapy or cetuximab with radiotherapy for locally advanced head and neck cancer: a systematic review and meta-analysis of published studies," Oral Oncology, vol. 50, no. 11, pp. 1041-1048, 2014.

[4] M. Kong and S. E. Hong, "Tumor regression patterns based on follow-up duration in patients with head and neck squamous cell carcinoma treated with radiotherapy or chemoradiotherapy," Clinical and Experimental Otorhinolaryngology, vol. 8, no. 4, pp. 416-421, 2015.

[5] L. A. Torre, F. Bray, R. L. Siegel, J. Ferlay, J. Lortet-Tieulent, and A. Jemal, "Global cancer statistics, 2012," CA: A Cancer Journal for Clinicians, vol. 65, no. 2, pp. 87-108, 2015.

[6] J. D. Suh and J. H. Cho, "Trends in head and neck cancer in South Korea between 1999 and 2012," Clinical and Experimental Otorhinolaryngology, vol. 9, no. 3, pp. 263-269, 2016.

[7] A. Argiris, M. V. Karamouzis, D. Raben, and R. L. Ferris, "Head and neck cancer," The Lancet, vol. 371, no. 9625, pp. 1695-1709, 2008.

[8] N. Denaro, M. C. Merlano, and E. G. Russi, "Follow-up in head and neck cancer: do more does it mean do better? A systematic review and our proposal based on our experience," Clinical and Experimental Otorhinolaryngology, vol. 9, no. 4, pp. 287297, 2016.

[9] D. Hanahan and R. A. Weinberg, "Hallmarks of cancer: the next generation," Cell, vol. 144, no. 5, pp. 646-674, 2011.

[10] J. D. Hayes and A. T. Dinkova-Kostova, “The Nrf2 regulatory network provides an interface between redox and intermediary metabolism," Trends in Biochemical Sciences, vol. 39, no. 4, pp. 199-218, 2014.

[11] G. M. DeNicola, F. A. Karreth, T. J. Humpton et al., "Oncogene-induced Nrf2 transcription promotes ROS detoxification and tumorigenesis," Nature, vol. 475, no. 7354, pp. 106-109, 2011.

[12] M. Diehn, R. W. Cho, N. A. Lobo et al., "Association of reactive oxygen species levels and radioresistance in cancer stem cells," Nature, vol. 458, no. 7239, pp. 780-783, 2009.

[13] R. A. Cairns, I. S. Harris, and T. W. Mak, "Regulation of cancer cell metabolism," Nature Reviews Cancer, vol. 11, no. 2, pp. 85-95, 2011.

[14] C. Gorrini, I. S. Harris, and T. W. Mak, "Modulation of oxidative stress as an anticancer strategy," Nature Reviews Drug Discovery, vol. 12, no. 12, pp. 931-947, 2013.

[15] A. Glasauer and N. S. Chandel, "Targeting antioxidants for cancer therapy," Biochemical Pharmacology, vol. 92, no. 1, pp. 90-101, 2014.

[16] B. X. Liu, J. Y. Zhou, Y. Li et al., "Hederagenin from the leaves of ivy (Hedera helix L.) induces apoptosis in human LoVo colon cells through the mitochondrial pathway," BMC Complementary and Alternative Medicine, vol. 14, no. 1, p. 412, 2014.

[17] Y. Gao, C. He, W. Bi, G. Wu, and E. Altman, "Bioassay guided fractionation identified hederagenin as a major cytotoxic agent from Cyclocarya paliurus leaves," Planta Medica, vol. 82, no. 1-2, pp. 171-179, 2016.

[18] J. Wang, X. Z. Zhao, Q. Qi et al., "Macranthoside B, a hederagenin saponin extracted from Lonicera macranthoides and its anti-tumor activities in vitro and in vivo," Food and Chemical Toxicology, vol. 47, no. 7, pp. 1716-1721, 2009.

[19] D. Rodriguez-Hernandez, A. J. Demuner, L. C. Barbosa, R. Csuk, and L. Heller, "Hederagenin as a triterpene template for the development of new antitumor compounds," European Journal of Medicinal Chemistry, vol. 105, pp. 57-62, 2015.

[20] A. Gepdiremen, V. Mshvildadze, H. Suleyman, and R. Elias, "Acute anti-inflammatory activity of four saponins isolated from ivy: alpha-hederin, hederasaponin- $\mathrm{C}$, hederacolchiside$\mathrm{E}$ and hederacolchiside- $\mathrm{F}$ in carrageenan-induced rat paw edema," Phytomedicine, vol. 12, no. 6-7, pp. 440-444, 2005.

[21] M. O. Hengartner, "The biochemistry of apoptosis," Nature, vol. 407, no. 6805, pp. 770-776, 2000.

[22] M. B. Sporn and K. T. Liby, "NRF2 and cancer: the good, the bad and the importance of context," Nature Reviews Cancer, vol. 12, no. 8, pp. 564-571, 2012.

[23] T. Ohta, K. Iijima, M. Miyamoto et al., "Loss of Keap1 function activates Nrf2 and provides advantages for lung cancer cell growth," Cancer Research, vol. 68, no. 5, pp. 1303-1309, 2008.

[24] L. M. Solis, C. Behrens, W. Dong et al., "Nrf2 and Keap1 abnormalities in non-small cell lung carcinoma and association with clinicopathologic features," Clinical Cancer Research, vol. 16, no. 14, pp. 3743-3753, 2010.

[25] A. Ohkoshi, T. Suzuki, M. Ono, T. Kobayashi, and M. Yamamoto, "Roles of Keap1-Nrf2 system in upper aerodigestive tract carcinogenesis," Cancer Prevention Research, vol. 6, no. 2, pp. 149-159, 2013.

[26] J. M. Cho, S. Manandhar, H. R. Lee, H. M. Park, and M. K. Kwak, "Role of the Nrf2-antioxidant system in cytotoxicity mediated by anticancer cisplatin: implication to cancer cell resistance," Cancer Letters, vol. 260, no. 1-2, pp. 96-108, 2008.

[27] X. J. Wang, Z. Sun, N. F. Villeneuve et al., "Nrf2 enhances resistance of cancer cells to chemotherapeutic drugs, the dark side of Nrf2," Carcinogenesis, vol. 29, no. 6, pp. 1235-1243, 2008.

[28] E. H. Kim, H. Jang, D. Shin, S. H. Baek, and J. L. Roh, “Targeting Nrf2 with wogonin overcomes cisplatin resistance in head and neck cancer," Apoptosis, vol. 21, no. 11, pp. 1265-1278, 2016.

[29] P. L. Lin, J. T. Chang, D. W. Wu, C. C. Huang, and H. Lee, "Cytoplasmic localization of Nrf2 promotes colorectal cancer with more aggressive tumors via upregulation of PSMD4," Free Radical Biology \& Medicine, vol. 95, pp. 121-132, 2016.

[30] R. Faraonio, P. Vergara, D. Di Marzo et al., "p53 suppresses the Nrf2-dependent transcription of antioxidant response genes," The Journal of Biological Chemistry, vol. 281, no. 52, pp. 39776-39784, 2006.

[31] L. Raj, T. Ide, A. U. Gurkar et al., "Selective killing of cancer cells by a small molecule targeting the stress response to ROS," Nature, vol. 475, no. 7355, pp. 231-234, 2011.

[32] J. L. Roh, E. H. Kim, J. Y. Park, J. W. Kim, M. Kwon, and B. H. Lee, "Piperlongumine selectively kills cancer cells and increases cisplatin antitumor activity in head and neck cancer," Oncotarget, vol. 5, no. 19, pp. 9227-9238, 2014.

[33] S. Homma, Y. Ishii, Y. Morishima et al., "Nrf2 enhances cell proliferation and resistance to anticancer drugs in human lung 
cancer," Clinical Cancer Research, vol. 15, no. 10, pp. 34233432, 2009.

[34] G. J. Kim, D. H. Song, H. S. Yoo, K. H. Chung, K. J. Lee, and J. H. An, "Hederagenin supplementation alleviates the proinflammatory and apoptotic response to alcohol in rats," Nutrients, vol. 9, no. 1, 2017.

[35] A. G. Wu, W. Zeng, V. K. Wong et al., "Hederagenin and $\alpha$-hederin promote degradation of proteins in neurodegenerative diseases and improve motor deficits in MPTP-mice," Pharmacological Research, vol. 115, pp. 25-44, 2017. 


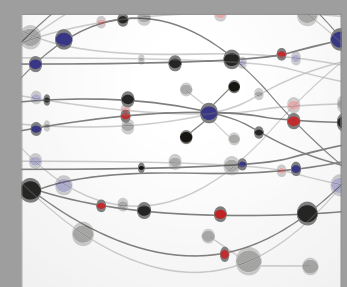

The Scientific World Journal
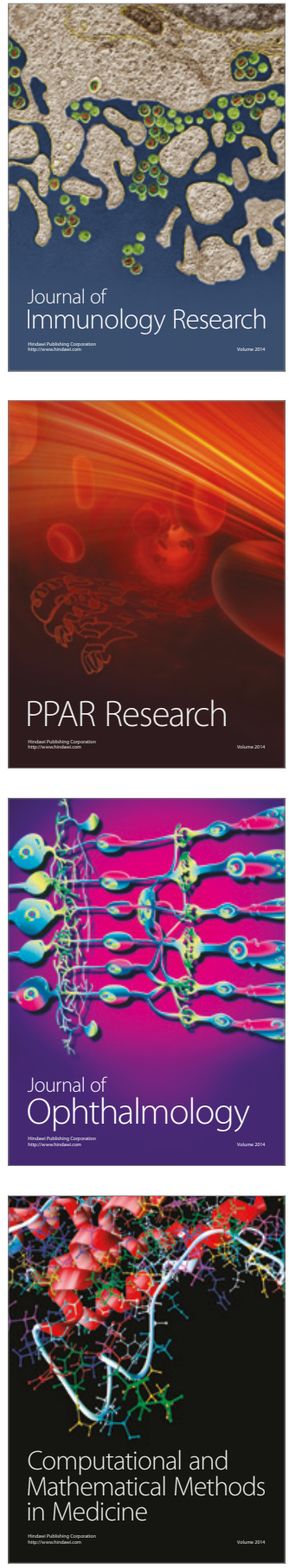

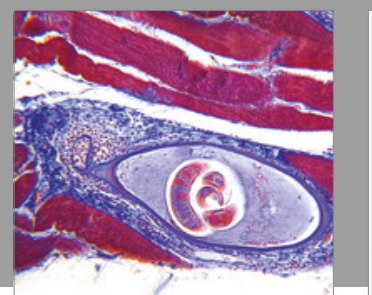

Gastroenterology Research and Practice
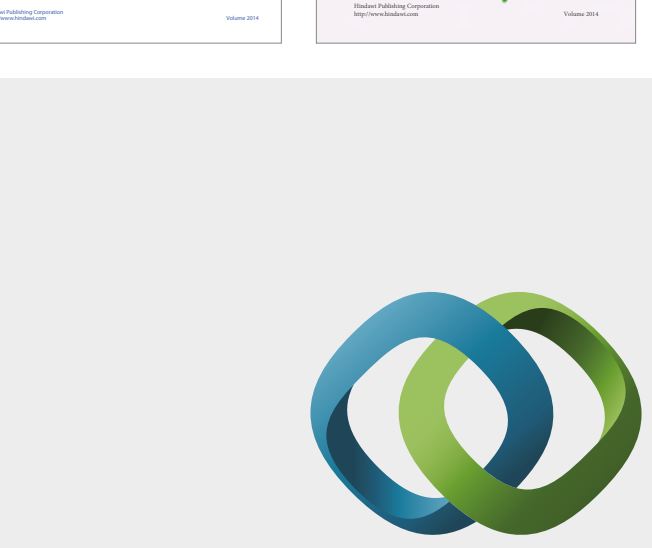

\section{Hindawi}

Submit your manuscripts at

https://www.hindawi.com
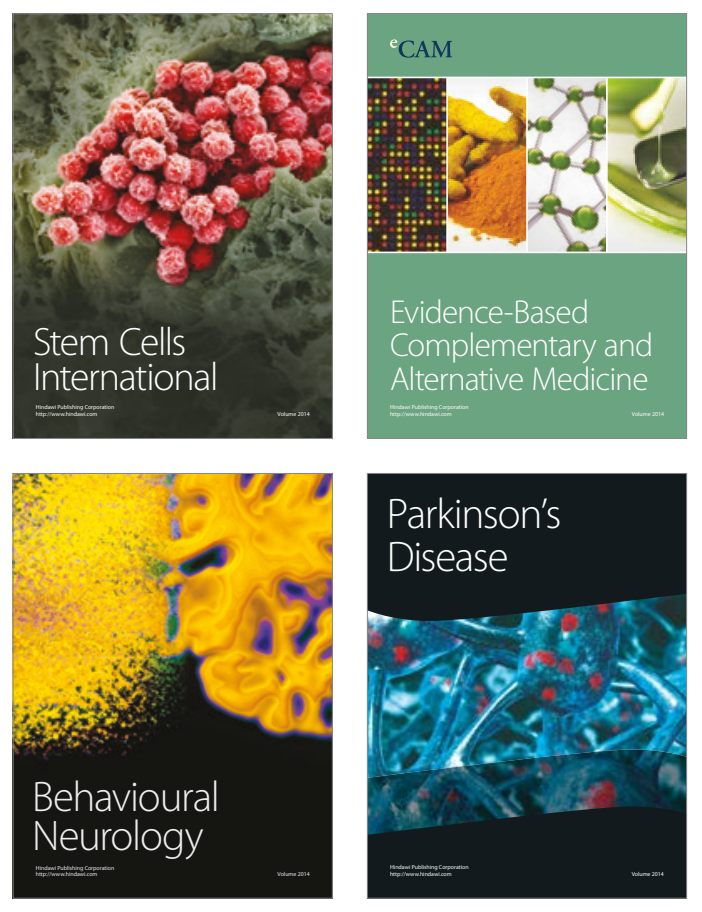
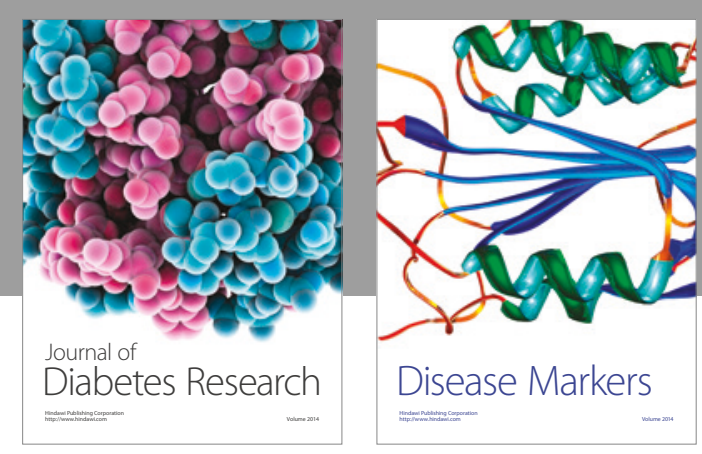

Disease Markers
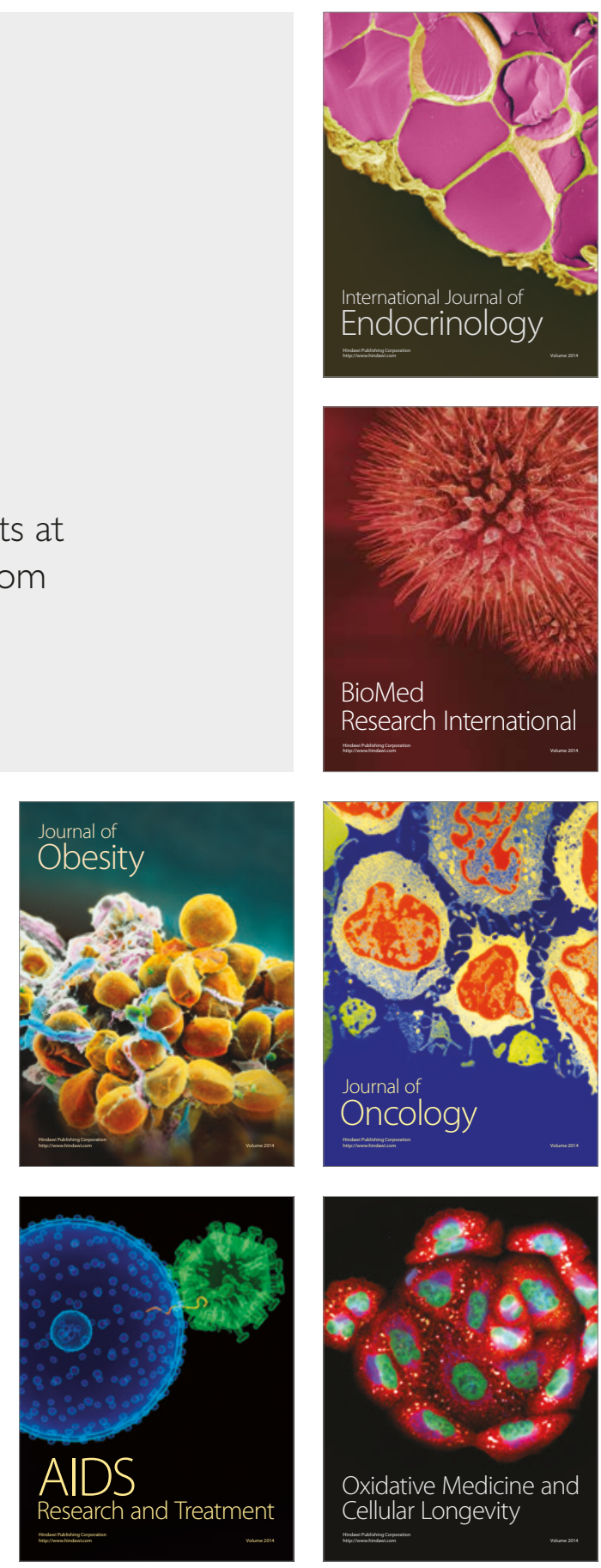